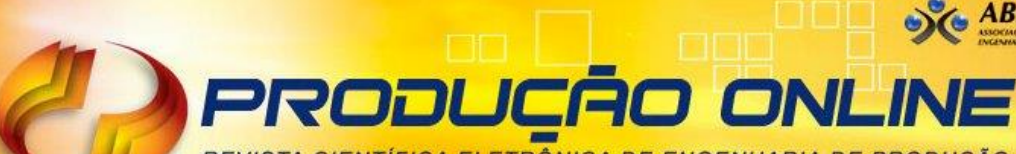 \\ REVISTA CIENTIFICA ELETRÔNICA DE ENGENHARIA DE PRODUÇÃO \\ ISSN 1676-1901
}

\section{ESTUDO LOGÍSTICO PARA INSTALAÇÃO DE NOVO CD: ABORDAGEM DIDÁTICA DE UM CASO REAL}

\section{LOGISTIC STUDY FOR INSTALLATION OF NEW CD: A DIDATIC APPROACH OF A REAL CASE}

\author{
Margareth Rodrigues de Carvalho Borella*E-mail: mrcborel@ucs.br \\ Guilherme Cardoso da Silva* E-mail: icborella@ucs.br \\ *Universidade de Caxias do Sul (UCS), Caxias do Sul, RS
}

Resumo: O objetivo desse trabalho é fazer um estudo logístico, utilizando técnicas qualitativas e métodos matemáticos, para definição da localização de um novo CD (Centro de Distribuição) e de sua operacionalização, por equipe própria ou de terceiros, de uma empresa distribuidora de componentes pneumáticos, localizada na cidade de Caxias do Sul, Rio Grande do Sul, Brasil. Foram utilizadas, observações in loco no atual CD da empresa para identificar as movimentações de transporte envolvidas, informações da internet sobre mapas rodoviários contendo as distâncias entre as localidades candidatas, dados de volumes transportados, áreas de armazenamento, custos por quilômetro rodado, registros e documentos da empresa. Foram utilizadas técnicas de localização industrial, cálculos de demanda futura e dados de custos logísticos na condução das análises de decisão. A alternativa mais viável envolve a instalação do novo CD na cidade de São Paulo, e as operações internas de movimentação, e externas para o atendimento aos clientes, em relação a esse $\mathrm{CD}$, devem ser conduzidas por equipe terceirizada na figura de operadores logísticos.

Palavras-chaves: Logística. Centro de Distribuição. Tomada de Decisão.

Abstract: The purpose of this work is to conduct a logistic study, using qualitative and quantitative methods for the installation of a new CD (Center of Distribution) and its operacionalization, by the own crew or the thirdy part, of a distribution company located in the city of Caxias do Sul, Rio Grande do Sul, Brazil. "In loco" observations were used in the current CD of the company to identify the transport movements present, information on the road maps containing the distances between the candidates places to implementation, transported volumes data, storage areas, costs per kilometer, records and company documents. Industrial localization techniques, future demand calculations and logistical costs data were used to conduct decision analysis. The most viable alternative is the installation of the CD in the city of São Paulo, and that internal operations and external customer service in relation to this CD should be conducted by outsourced personnel in the figure of logistics operators.

Keywords: Logistics. Center of Distribution. Making Decision. Logistic costs. Geographic location.

\section{INTRODUÇÃO}

Em um país com dimensões continentais como o Brasil, é muito importante ter uma boa estratégia logística, a fim de não gerar um gargalo no fornecimento dos produtos. Uma solução para facilitar as operações é a implantação de Centros de Distribuição $(C D)$ em pontos estratégicos, principalmente quando se trata de 
importação de produtos, visando estar próximo dos principais clientes, garantindo agilidade no recebimento e na entrega dos produtos, redução nos custos de distribuição, além de ter a possibilidade de obter incentivos fiscais. Conforme Nogueira (2012), a armazenagem está entre os tópicos mais importantes da logística, pois através dela é possível otimizar espaços e baixar os custos, proporcionando um produto mais barato para o mercado e garantindo o aumento das vendas. A questão que se pretende responder ao realizar esse trabalho é: Qual é a melhor alternativa de decisão, envolvendo a localização e a operacionalização, ou por equipe própria, ou equipe terceirizada, de um novo $C D$ de distribuidora de marcas nacionais e importadas de ferramentas e pneumáticos para a indústria metal-mecânica, automotiva, moveleira, calçadista, alimentício, refrigeração, entre outros?

Para responder a essa pergunta, o artigo está estruturado em um capítulo de revisão bibliográfica, focada em métodos e técnicas logísticas de localização geográfica, cálculo de previsão de demanda e de custos de estoques, alinhado com a temática empírica desse artigo. Na sequência é apresentado um capítulo sobre a metodologia utilizada nesse trabalho, contendo as etapas da pesquisa. O terceiro capítulo é constituído pela apresentação dos resultados com base nas técnicas apresentadas na revisão bibliográfica, analisando cada localidade candidata para sediar o novo $C D$, e por último, as considerações finais do trabalho com a discussão da alternativa mais viável, qualitativamente e quantitativamente, que consiste em instalar o CD na cidade de São Paulo, Brasil, e operacionalizada por equipe terceirizada, na figura de operador logístico. O custo anual das novas instalações, traduzido como investimento, é estimado em $R \$ 275.800,00$, que corresponde a $10,64 \%$ do faturamento anual da empresa, e que proporcionará $15 \%$ de redução de custo com as operações logísticas terceirizadas, no que se refere a aluguéis, manutenções prediais, equipamentos utilizados, encargos trabalhistas entre outros.

\section{TÉCNICAS PARA LOCALIZAÇÃO DE CENTROS DE DISTRIBUIÇÃO}

\subsection{Aspectos determinantes}

Para Gurgel (2008) a decisão sobre a mudança ou a abertura de um novo CD tem caráter irreversível por um prazo de 10 a 20 anos, e por isso não pode ser tomada 
com base unicamente na situação atual da empresa, deve-se também levar em consideração a projeção da situação futura.

Conforme Corrêa (2012) diante da globalização atual, a decisão para a escolha da localização de um CD é mais complexa do que nunca, os principais aspectos que levam a se pensar nesta mudança incluem: desempenho logístico, alterações na malha de transporte, mudança na localização de mercados fornecedores ou consumidores de produtos, pressões competitivas por redução de custo. $E$ as considerações referentes ao ambiente físico e de negócios, incluem aspectos como preço, incentivos fiscais, zonas livres de comércio, legislação, impactos ambientais, acesso a infraestrutura entre outros.

\subsection{Métodos para a localização}

A literatura, utilizada em sala de aula e pelas empresas de consultoria, descreve alguns métodos para avaliação do melhor local para a instalação de uma empresa, fábrica ou CD, envolvendo técnicas qualitativas e quantitativas demonstradas na sequência. Ainda há pouca literatura científica utilizando abordagens de otimização multicritério, a partir de técnicas de modelagem e pesquisa operacional (Guazzelli e Cunha, 2015), o que levou, nesse estudo, a optar por uma revisão bibliográfica mais objetiva e prática.

\subsubsection{Método de Ponderação de Fatores}

Conforme Corrêa e Corrêa (2012) e Gurgel (2008) o método de ponderação de fatores é um método subjetivo, que envolve confrontar e avaliar diversas alternativas de macrolocalizações, ponderando fatores locacionais. O método consiste em atribuir pesos para fatores, como por exemplo, restrições ambientais, disponibilidade de mão de obra, sistema de transportes, proximidade de mercados, qualidade de vida, proximidade de matérias primas e outros, que ao serem somados devem totalizar 100\% (Heizer e Render, 2001). Para cada um desses aspectos é dado uma nota de um a cinco, a pior e melhor condição, respectivamente, presente em cada localidade candidata à instalação. O maior escore revela o local mais adequado para a instalação. 


\subsubsection{Método Carga Distância}

De acordo com Krajewski et al. (2009) o método segue um modelo matemático, utilizado para avaliar determinadas localizações, tendo por referência aspectos de proximidade. Seu principal objetivo é selecionar qual localização que minimiza a soma de todas as cargas (Gaither e Frazier, 2002), multiplicado pelo tempo ou a distância percorrida.

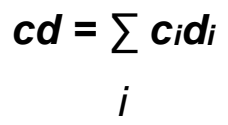

Onde:

$c d=$ carga distância

$c_{i}=$ cargas

$d_{i}=$ distância ou tempo percorrido

\subsubsection{Método do Ponto de Equilíbrio}

Krajewski et al. (2009) afirmam que a análise do ponto de equilíbrio compara alternativas de localização, tendo por direção fatores quantitativos que podem ser expressos em valores de custo total. Este método é muito utilizado quando se pretende definir faixas nas quais cada alternativa torna-se a ideal de acordo com a quantidade armazenada ou produzida e seus respectivos custos totais (Moreira, 2008).

\subsubsection{Método do Centro de Gravidade}

Conforme Grant (2013) e Corrêa e Corrêa (2012), o método do centro de gravidade é uma metodologia de planejamento de cadeia de suprimentos, que tem como função localizar centrais de distribuição próximas aos locais das empresas, dos fornecedores e clientes para minimizar o custo com a logística de transporte. O método calcula as coordenadas utilizando a fórmula:

$$
C x=\frac{\sum \operatorname{dix} V i}{\sum V i} \text { e } C y=\frac{\sum \operatorname{diy} V i}{\sum V i}
$$

Onde:

$C x=$ coordenada $x$ (eixo horizontal) do centro de gravidade

$C y=$ coordenada y (eixo vertical) do centro de gravidade 
$y=$ coordenada y (eixo vertical) do centro de gravidade

$d i x=$ coordenada do iésimo local

diy $=$ coordenada y iésimo local

$V i=$ volume de bens movimentados para ou do iésimo local

\subsection{Dimensionamento do estoque}

Para Dias (2012) a gestão de estoque é traçada com base na previsão de consumo dos produtos, que possibilita estabelecer as estimativas futuras sobre as quantidades que serão compradas pelos clientes ao longo da cadeia de suprimentos, e consequentemente deverão estar armazenadas nos CDs para atender às cadeias de distribuição (Taylor, 2005). Um bom processo de tomada de decisão sobre recursos que tenham inércia decisória baseia-se numa visão de futuro com uma boa estimação, obtida a partir das previsões (Corrêa, 2014).

\subsubsection{Método da Média Móvel}

Segundo Corrêa e Corrêa (2012), o modelo de média móvel assume que o melhor cenário do futuro é dado pela média dos últimos períodos. Para Dias (2012) a previsão deste modelo é geralmente menor que os valores ocorridos se o padrão de consumo for crescente, e ao contrário se o padrão for decrescente. A fórmula é dada por:

$$
\mathrm{CM}=\frac{\mathrm{C}_{1}+\mathrm{C}_{2}+\ldots \ldots+\mathrm{C}_{N}}{\mathrm{~N}}
$$

Onde:

$\mathrm{CM}=$ consumo médio

$\mathrm{C}_{1}$ a $\mathrm{C}_{\mathrm{N}}=$ consumo nos períodos anteriores

$\mathrm{N}$ = número de períodos

\subsubsection{Método da Média Móvel Ponderada}

Este método baseia-se na ponderação de cada período anterior para determinar a previsão do próximo período (Russo, 2013). No estudo de Cecatto e 
Belfiori (2015), a média móvel e móvel ponderada ainda estão entre os métodos utilizados nas indústrias alimentícias. A ponderação é feita atribuindo pesos maiores aos períodos mais recentes e pesos menores aos períodos mais distantes do período de previsão. A soma dos pesos deve totalizar $100 \%$. A sensibilidade e o conhecimento do mercado pelos gestores são fundamentais. A formula é dada por:

$$
D P(m m p)=\left(C_{1} \times P_{1}\right)+\left(C_{2} \times P_{2}\right)+\left(C_{3} \times P_{3}\right)+\ldots+\left(C_{n} \times P_{n}\right)
$$

Onde:

$\mathrm{DP}(\mathrm{mmp})=$ demanda prevista pela média móvel ponderada

$\mathrm{C}_{1}$ a $\mathrm{C}_{\mathrm{n}}=$ consumo real nos períodos anteriores

$\mathrm{P}_{1}$ a $\mathrm{P}_{\mathrm{n}}=$ ponderação atribuída a cada período, conforme a sensibilidade do gestor.

\subsubsection{Método da Média Exponencial com Ponderação}

Esse método elimina muitas desvantagens dos métodos da média móvel e da média móvel ponderada (Dias, 2012). Para se gerar a previsão para o próximo período, apenas três valores são necessários, a previsão do último período, o consumo real desse último período, e uma constante que determina valor ou ponderação atribuída aos valores mais recentes.

Russo (2013) complementa que essa constante, cujo símbolo é representado pelo alfa $(\alpha)$, pode variar entre 0 e $1(0 \leq \alpha \leq 1)$. As organizações adotam valores para a entre 0,1 a 0,3. A próxima previsão é igual à previsão anterior mais a constante de amortecimento, multiplicado pelo erro de previsão, conforme a fórmula:

$\overline{C_{t}}=\overline{C_{t-1}}+\alpha \cdot\left(C_{t-1}-\overline{C_{t-1}}\right)$ para $0 \leq \alpha \leq 1$

Onde:

$\overline{\mathrm{Ct}}=$ previsão de consumo para o próximo período

$\overline{\mathrm{Ct}}-1$ = previsão de consumo para o período passado

$\mathrm{Ct}-1=$ consumo efetivo no período passado

$\alpha=$ coeficiente de ajustamento 


\subsubsection{Método dos Mínimos Quadrados}

Conforme Dias (2012), este método é utilizado para determinar a melhor linha de ajuste, passando mais próximo de todos os dados coletados, ou seja, minimiza as diferenças entre os dados observados de um modelo de consumo linear. Para Russo (2013) este é um dos modelos que mais favorece a precisão das previsões, uma vez que é uma contínua aproximação do consumo real. O método se baseia na equação da reta $(y=a+b . x)$.

Onde:

$\mathrm{y}=$ valor da variável dependente

$\mathrm{a}=$ intercepto de y

$\mathrm{b}=$ declive da linha

$\mathrm{x}=\mathrm{a}$ variável independente

\subsection{Custos dos estoques}

Os custos logísticos envolvem os custos com: transporte, armazenagem, estoque, portuários, tributários e de transbordo (Kussano e Batalha, 2012). Considerando a armazenagem, a atividade principal de um CD, duas variáveis acabam aumentando estes custos, a quantidade de produtos estocados e o tempo de permanência em estoque. Todos estes custos relacionados acabam recebendo o nome de custo de armazenagem, os mesmos são calculados baseando-se no estoque médio e geralmente indicados em forma de porcentagem do valor em estoque (Dias, 2012).

\subsubsection{Custo de Armazenagem}

Entre os vários tipos de custos que afetam a rentabilidade das empresas, está o custo de armazenagem (Dias, 2012). O cálculo é dado pela seguinte expressão:

$$
C a=\left[\begin{array}{l}
Q \\
2
\end{array}\right] \cdot T . P . I
$$

Onde: 
$\mathrm{Ca}=$ custo unitário de armazenagem

$Q=$ quantidade de material em estoque no período considerado

$\mathrm{P}=$ preço unitário do material

$I$ = taxa de armazenamento (\% do custo unitário)

$\mathrm{I}=\mathrm{la}+\mathrm{lb}+\mathrm{Ic}+\mathrm{Id}+\mathrm{le}+\mathrm{If}$

$\mathrm{T}=$ tempo considerado no custo total da armazenagem

la = taxa de retorno de capital; lb = taxa de armazenamento físico; lc = taxa de seguro; ld = taxa de movimentação, manuseio e distribuição de material; le = taxa de obsolescência, If = outras taxas, como água, luz, etc.

\subsubsection{Custo de Pedido}

Conforme Dias (2012) para calcular o custo anual de todos os pedidos de um período, é necessário calcular o custo de cada pedido e multiplicar pelo número de vezes que foi efetuado no período estipulado. Este custo pode ser calculado através da expressão:

$\mathrm{CTP}=\mathrm{B} \times \mathrm{N}$

Onde:

CTP $=$ custo total anual de pedidos

$\mathrm{B}=$ custo unitário de pedido

$\mathrm{N}$ = número de pedidos efetuados durante um ano

\section{MÉTODO DE PESQUISA}

Para Diehl (2004) uma pesquisa constitui-se em um procedimento racional e sistemático, no qual o principal objetivo é proporcionar respostas aos problemas propostos e oportunidades de melhorias identificadas. $\mathrm{O}$ autor complementa que neste contexto, a metodologia pode ser definida como o estudo e a avaliação dos muitos métodos, com o intuito de identificar limitações e possibilidades no âmbito de sua aplicação.

Conforme Roesch (2013) existem cinco tipos de metodologia para a realização de trabalhos de conclusão, conforme com os objetivos que se pretende atingir:

a) Pesquisa aplicada: gerar soluções potenciais para os problemas humanos; 
b) Avaliação de resultados: julgar a efetividade de um plano ou programa;

c) Avaliação formativa: melhorar um programa ou plano, acompanhando sua implantação;

d) Pesquisa-diagnóstico: explorar o ambiente organizacional e de mercado, levantar e definir problemas;

e) Proposição de planos: apresentar soluções para problemas já diagnosticados.

Esse trabalho é de natureza exploratória com características de pesquisadiagnóstico e baseada num estudo de caso de uma empresa nacional, cuja matriz está localizada na cidade de Caxias do Sul, estado do Rio Grande do Sul, Brasil, estando o seu negócio baseado predominantemente na aquisição e comercialização de componentes e peças para pneumáticos e refrigeração, para fins industriais.

Os objetivos desse trabalho estão relacionados com o estudo da localização mais adequada para um novo armazém que irá distribuir os produtos para as revendas e distribuidores clientes da empresa alvo desse estudo. As etapas necessárias para atingir os objetivos são:

- Em primeiro lugar é realizado um levantamento dos aspectos mais relevantes a serem considerados neste estudo, detalhamento sobre as alternativas de localidade e análise comparativa baseadas na revisão bibliográfica.

- Em segundo lugar, após os resultados da análise comparativa, são identificadas as principais vantagens das localidades avaliadas, definição da localidade escolhida e comparação com o modo atual de armazenagem e distribuição, a fim de verificar o possível ganho nas operações.

- Em terceiro lugar é realizada uma análise que identifique qual modo de investimento é mais viável, alugar um pavilhão para instalar este novo $C D$ ou terceirizar as operações.

Para que essas etapas fossem realizadas, tratamento matemático, quantitativo e qualitativo foi dado nas diversas fases da análise sobre os possíveis locais para a instalação do novo CD. Dados como: quantidades movimentadas, quantidades recebidas, quantidades armazenadas, trajetos percorridos, distâncias entre origem e destinos, vantagens qualitativas de acesso, qualidade da mão-de-obra, infraestrutura, segurança e outros. Orçamento sobre custos de movimentação, armazenagem, 
expedição e seguro por unidade estocada, conduzida por operadores logísticos, foi comparado com os custos fixos dessa operacionalização pela própria empresa.

A definição dos critérios e fatores qualitativos, relevantes à instalação do novo CD, bem como as pontuações e classificações, normalmente marcadas pela subjetividade, foi conduzida por meio de discussão com os dois diretores e o profissional de logística. $O$ consenso foi alcançado rapidamente devido a predominância de igualdade de opiniões entre esses gestores, uma vez que o problema já vinha sendo debatido há algum tempo pela empresa.

\section{APRESENTAÇÃO DOS RESULTADOS}

Neste capítulo é apresentada a estrutura da empresa, a armazenagem e a distribuição dos produtos. Em seguida é realizado um estudo sobre melhor opção para a implantação de um novo CD e as possíveis vantagens dessa mudança.

\subsection{Estrutura atual de armazenamento e distribuição}

A empresa possui na sua linha de produtos em torno de 15 mil itens, divididos em vários segmentos específicos, como refrigeração, abrasivos e usinagem. A maioria destes itens é de origem importada, tendo todo seu processo de embarque e despacho terceirizado através de despachantes aduaneiros. $O$ recebimento destes produtos no país ocorre através do porto marítimo de Rio Grande, no estado do Rio Grande do Sul, após eles são transferidos para o porto seco de Caxias do Sul para desembaraço fiscal, culminando na entrega da carga na empresa.

A matriz da empresa conta com 50 funcionários e com uma área construída de $3.000 \mathrm{~m}^{2}$, sendo dividida em $2.300 \mathrm{~m}^{2}$ destinados a armazenagem, recebimento e expedição de materiais e $700 \mathrm{~m}^{2}$ destinados à área administrativa e de operações. Atualmente a matriz funciona como um centro de distribuição, onde todos os produtos adquiridos são recebidos armazenados e posteriormente distribuídos para as suas filiais e clientes espalhados pelo país. A empresa possui ainda três filiais, em Novo Hamburgo e Erechim, ambas no estado do Rio Grande do Sul, totalizando 10 funcionários e uma no estado de Santa Catarina, contando com três funcionários. A empresa iniciou suas atividades no ano de 2000, e mesmo sendo relativamente jovem 
conquistou a certificação ISO 9001/2000 no ano de 2006. A empresa conta ainda com 14 representantes comerciais fornecendo produtos para os setores industriais, metalmecânico, calçados, refrigeração e outros. São atendidos aproximadamente 10.000 clientes, em que 5\% apresentam um potencial poder de compra e que estão localizados em sua maioria nos estados do Rio de Janeiro, São Paulo e Rio Grande do Sul.

A empresa trabalha com materiais de alta rotatividade, e por isso optou em trabalhar com porta pallets e prateleiras. Com esse método foi possível utilizar o espaço destinado a armazenagem com melhor aproveitamento, utilizando o espaço horizontal e o vertical, aumentado a área útil. Outras vantagens obtidas desse método são: acesso simplificado aos materiais, agilidade na hora da separação e baixa manutenção na estrutura de armazenamento.

Quanto ao gerenciamento do estoque a empresa utiliza o WMS (Warehouse Management System), através do qual são controladas e administradas todas as operações de estoque: recebimento, endereçamento de localização, armazenagem, separação dos pedidos, expedição e despacho dos materiais. Depois dos dados coletados é realizada uma sincronização, por transmissão em tempo real, através de redes sem fio, com o banco de dados do WMS, o que possibilita extrair relatórios úteis sobre o status das mercadorias no estoque, relação de produtos vendidos, entre outras informações para a tomada de decisão.

\subsection{Análise das alternativas de decisão para o novo cd}

A empresa possui desvantagens comerciais perante seus concorrentes devido à localização atual de seu CD, ao prazo de entrega estendido e ao custo com transporte. Evidencia-se a necessidade de mudança de localização de seu CD, o qual possibilitará melhorar o nível do serviço prestado, aumentando a competitividade perante o mercado. Para instalar esse novo CD foram definidos os seguintes objetivos:

a) Identificar as localidades que são mais vantajosas para implantação do novo CD.

b) Analisar as vantagens da operação nessas localidades, estimando os ganhos. 
c) Avaliar a melhor alternativa visando a instalação do novo CD.

Para determinar as localidades, foi realizada uma lista dos aspectos mais importantes considerados pela empresa para implantação do novo centro de distribuição, divididos em principais e secundários, conforme Quadro 1.

Quadro 1 - Aspectos principais e secundários na escolha da localidade

\begin{tabular}{|l|l|}
\hline \multicolumn{1}{|c|}{ PRINCIPAIS } & \multicolumn{1}{|c|}{ SECUNDÁRIOS } \\
\hline Proximidade do mercado & Malhas de transporte \\
\hline $\begin{array}{l}\text { Facilidade de acesso aos fornecedores, regiões com } \\
\text { portos. }\end{array}$ & Proximidade dos principais concorrentes \\
\hline & Mão de obra \\
\hline
\end{tabular}

No Ano de 2016 a empresa faturou aproximadamente 30 milhões de reais e a Figura 1 apresenta o percentual desse total em cada região do Brasil.

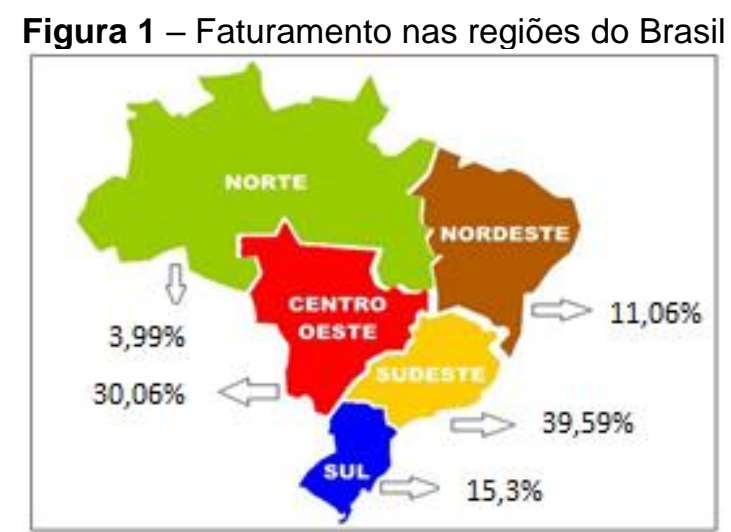

Fonte: Elaborada pelo autor com base em informações do setor financeiro (2016)

Quanto à facilidade de acesso aos principais fornecedores, é possível afirmar que eles estão centralizados na região Sudeste, mais precisamente no estado de São Paulo. Outro fator que foi levado em consideração é sobre os produtos importados, que representam a maioria dos produtos comercializados pela empresa. Em função destes aspectos foram levantados quais são os principais portos nas regiões com maior volume de vendas (Figura 2). 
Figura 2 - Principais portos nas regiões, sul, sudeste do Brasil

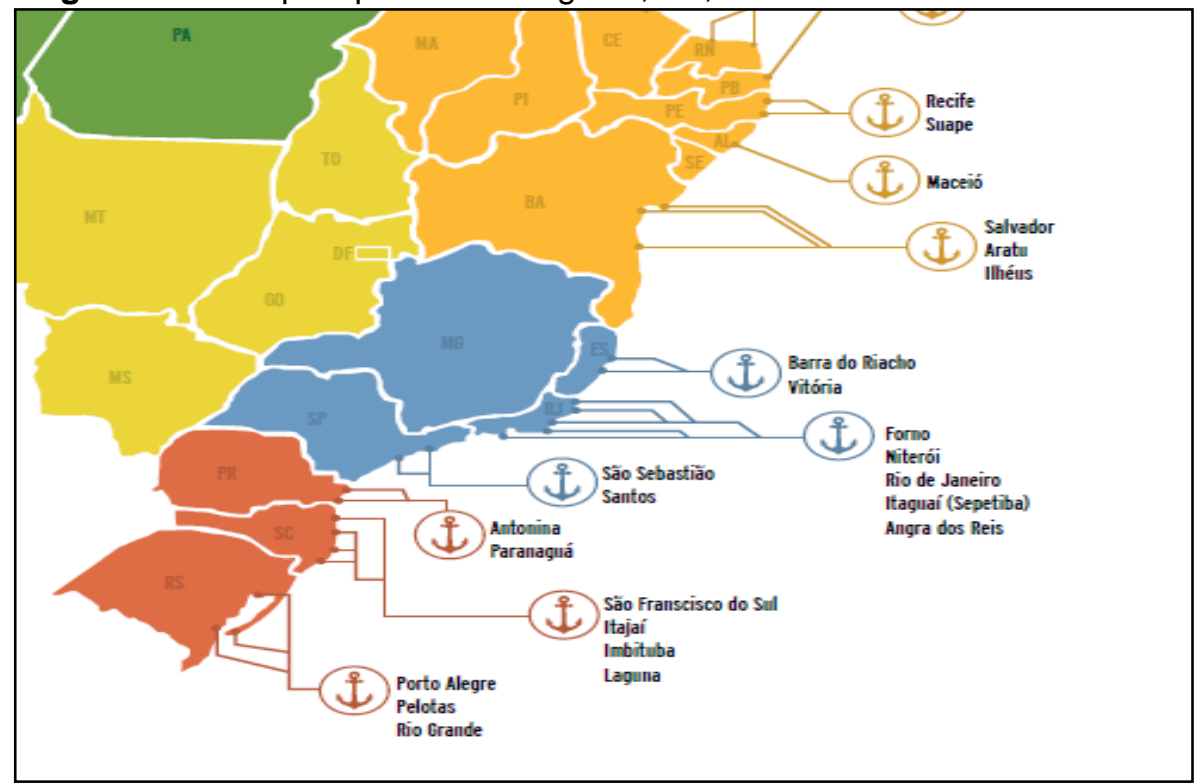

Fonte: Adaptado de Antaq (2016)

Quanto às malhas de transporte é possível qualitativamente dizer que:

- O sudeste é uma região privilegiada, existem boas rodovias, principalmente no estado de São Paulo, muitas delas autoestradas com pistas duplas. O transporte marítimo é grande, destacando-se os portos de Tubarão (Vitória), Rio de Janeiro e Santos que é o maior da América Latina. O transporte aéreo é qualificado, possui os mais modernos aeroportos do país, já o transporte fluvial, apesar de existir, acontece em menor volume.

- Na região sul há uma boa malha rodoviária, apesar de insuficiente pela grande movimentação de carga leve e pesada. O transporte fluvial é bom em alguns rios e o marítimo se destaca no porto de Paranaguá (Paraná), com grande movimento, principalmente para a exportação. Existe um razoável movimento ferroviário apesar do Brasil ser bastante deficiente neste modal, já o transporte aéreo é bem movimentado, principalmente em Porto Alegre, Curitiba, Florianópolis, Foz do Iguaçu, Londrina e Itajaí.

- No Centro-oeste, os principais centros urbanos da região são Brasília, Goiânia, Campo Grande, Cuiabá, Dourados e Anápolis. O estado de Goiás possui a segunda melhor e mais conservada malha rodoviária do país, perdendo apenas para São Paulo. Possui tráfego aéreo intenso, já no modo fluvial existe um movimento razoável nos estados de Mato Grosso e Mato Grosso do Sul. 
Quanto à mão-de-obra, as corporações geralmente se encontram estabelecidas em grandes centros urbanos que aglomeram um grande contingente populacional. Caso a melhor opção esteja voltada para um centro de distribuição próprio, a empresa terá que contratar alguns profissionais com qualificação suficiente para coordenar todas as operações sem comprometer o nível de serviço prestado. Caso a escolha seja por terceirizar o armazenamento e a distribuição, toda a responsabilidade se volta para a empresa contratada, diminuindo os problemas relacionados com funcionários.

\subsubsection{Definição do local com maior potencial baseado nos tópicos anteriores}

Para definir quais estados fariam parte do próximo critério eliminatório foram considerados no processo decisório, a quantidade de clientes em potencial presente em cada estado da região sudeste, conforme Figura 3, bem como a presença de cidades portuárias para o recebimento dos produtos importados. Assim, as cidades pré-selecionadas foram São Paulo - SP, Rio de Janeiro - RJ e Vitória - ES. O estado de Minas Gerais não atende aos critérios de maior número de clientes e nem da presença de um porto marítimo para o desenvolvimento dos processos de importação.

Figura 3 - Estados da região sudeste com maior concentração de clientes

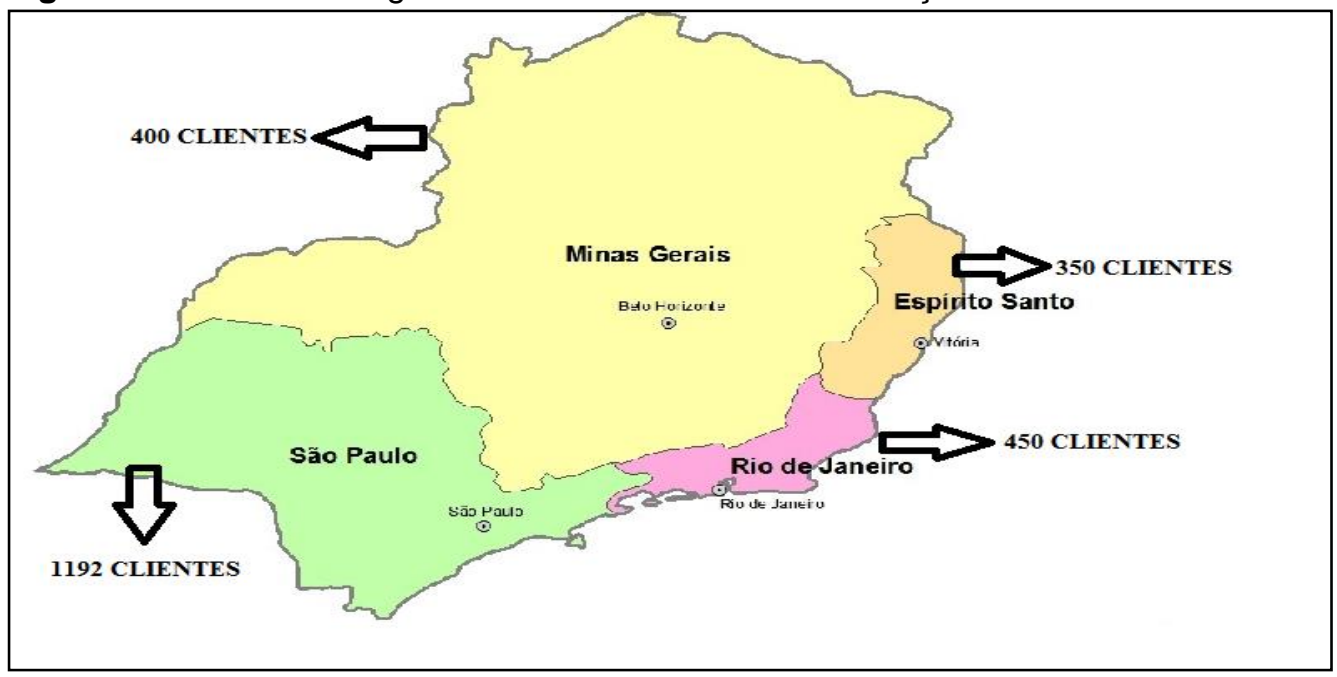

Fonte: Elaborada pelo autor com base em informações do setor comercial (2016) 


\subsubsection{Avaliação das cidades candidatas à instalação do $C D$}

O primeiro passo nesta etapa foi realizar comparações entre os locais específicos através dos métodos de avaliação para localização de unidades de operação, somados aos aspectos qualitativos.

A) Método Ponderação de Fatores

Para este método de avaliação foram selecionados os fatores mais determinantes para a localização, conforme a visão da empresa. A Tabela 1 envolve a classificação que varia de 1 (mais baixa ou ruim) até 5 (mais alta ou excelente). $A$ pontuação é obtida multiplicando-se a nota pelo peso de cada fator considerado. A cidade de São Paulo atingiu a maior pontuação totalizando 445, podendo ser considerada como a cidade mais atrativa para a instalação do novo CD.

Tabela 1 - Método da ponderação de fatores para as candidatas

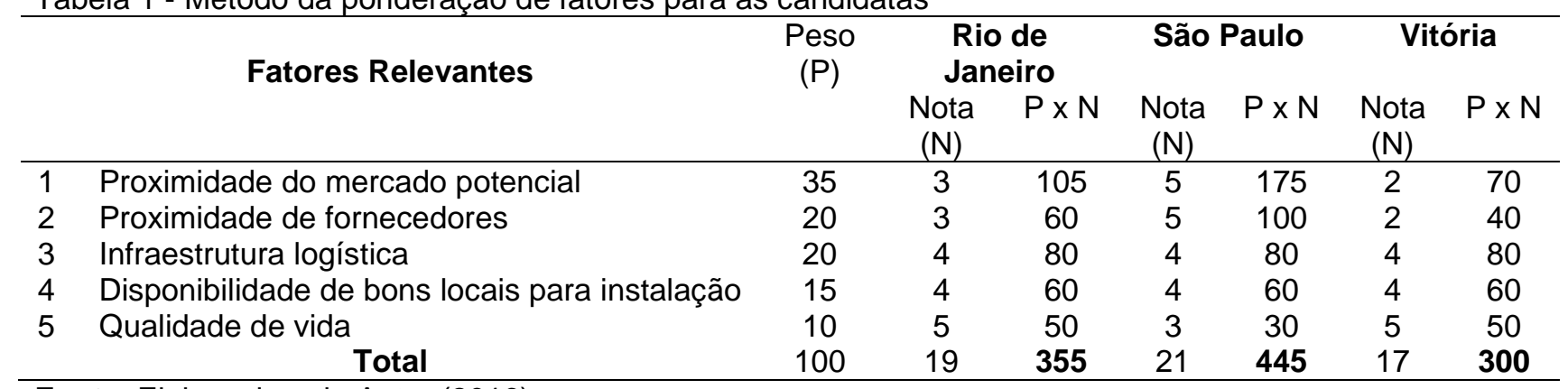

Fonte: Elaborada pelo Autor (2016)

\section{B) Método Carga-Distância}

Como a empresa tem o transporte terceirizado, este método traz informações que não seriam relevantes, uma vez que essa operação se manterá terceirizada. Mesmo assim, foi conduzido um levantamento sobre o volume de cargas, em toneladas, movimentado no ano de 2016, bem como o lead time de entrega (Tabela 2 e 3), considerando a estrutura atual, cuja origem é do CD da cidade de Caxias do Sul, junto com a estrutura futura, as cidades candidatas ao novo $C D$, que enviam cargas para os destinos em vários estados do Brasil. 
Tabela 2 - Toneladas transportadas ao longo de 2016

\begin{tabular}{cc}
\hline Período 2016 & Quantidade transportada (tonelada) \\
\hline Janeiro & 19.655 \\
Fevereiro & 22.512 \\
Março & 23.254 \\
Abril & 22.587 \\
Maio & 18.142 \\
Junho & 15.557 \\
Julho & 17.421 \\
Agosto & 18.882 \\
Setembro & 18.882 \\
Outubro & 20.998 \\
Novembro & 24.512 \\
Dezembro & 18.990 \\
Total & $\mathbf{2 4 1 . 3 9 2}$ \\
\hline
\end{tabular}

Fonte: Departamento logística da empresa (2016)

Pela Tabela 3, a cidade de São Paulo totalizaria o menor lead-time ao movimentar cargas ao longo do ano para os estados brasileiros listados.

Tabela 3 - Lead time de entrega em dias

\begin{tabular}{|c|c|c|c|c|c|}
\hline & Origem: Caxias do Sul & Origem: Rio de Janeiro & Origem: São Paulo & Origem: Vitória & Destinos \\
\hline$g$ & 1 & 4 & 3 & 4 & RS \\
\hline . & 2 & 3 & 2 & 3 & sc \\
\hline$\varepsilon$ & 2 & 4 & 2 & 4 & PR \\
\hline ฮิ & 3 & 1 & 1 & 3 & SP \\
\hline$\pi$ & 3 & 1 & 1 & 1 & RJ \\
\hline बे & 4 & 1 & 1 & 1 & MG \\
\hline \pm & 10 & 7 & 4 & 7 & BA \\
\hline ш & 14 & 9 & 7 & 9 & CE \\
\hline \% & 25 & 17 & 18 & 15 & $\mathbf{A M}$ \\
\hline & 6 & 3 & 3 & 3 & DF \\
\hline สํㄴ & 6 & 3 & 3 & 3 & GO \\
\hline ¿ँ & 6 & 3 & 3 & 3 & MT \\
\hline & 10 & 2 & 4 & 1 & ES \\
\hline Total & 92 & 58 & 52 & 57 & \\
\hline
\end{tabular}

Fonte: Transportadoras que prestam serviços para a empresa (2016)

C) Método do Centro de Gravidade

Para realizar a comparação dos locais específicos foi levantada a informação de volume de carga transportada em toneladas para os estados de SP, RJ e ES no ano de 2016, que foram, respectivamente, 28.124, 9.222 e 7.469 toneladas. Outro dado necessário foi o custo de transporte por tonelada, possibilitando assim conseguir as coordenadas que são necessárias para os cálculos, conforme Figura 4. 
Figura 4 - Grid para estudo locacional

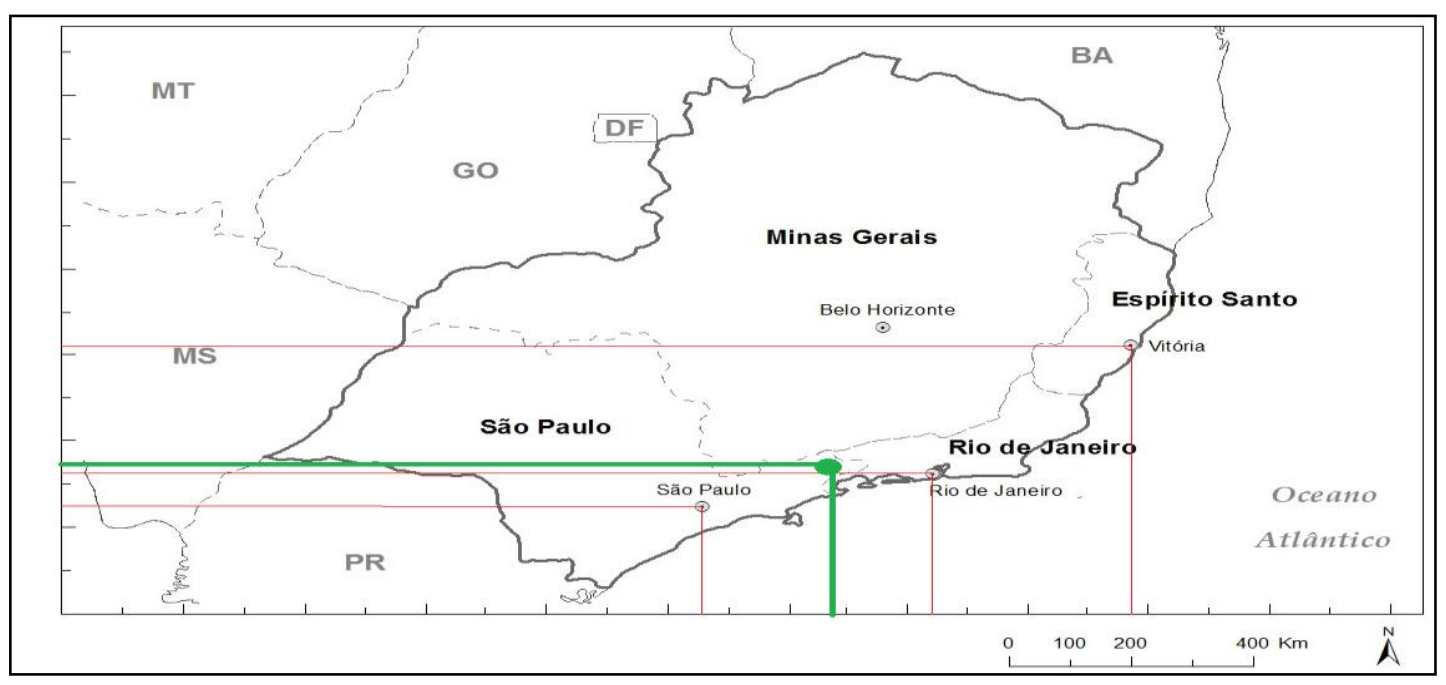

Fonte: Elaborada pelo autor a partir de base cartográfica do IBGE (2016)

A Tabela 4 apresenta as quantidades a serem transportadas e o custo do transporte conforme dados fornecidos pela transportadora, considerando os locais específicos como origem. Também foram acrescentadas as informações sobre as coordenadas obtidas pelo mapa.

Tabela 4 - Dados para o método do centro de gravidade

\begin{tabular}{lcccc}
\hline Local & $\begin{array}{c}\text { Quantidade } \\
\text { (toneladas com } \\
\text { base em 2016) }\end{array}$ & $\begin{array}{c}\text { Custos transporte } \\
\text { (R\$/tonelada/Km } \\
\text { rodado) }\end{array}$ & $\begin{array}{c}\text { Coordenada } \\
\text { horizontal X }\end{array}$ & $\begin{array}{c}\text { Coordenada } \\
\text { vertical Y }\end{array}$ \\
\hline São Paulo (SP) & 28.124 & 1,40 & 10,5 & 2,5 \\
Rio de Janeiro (RJ) & 9.222 & 1,90 & 14,2 & 3,1 \\
Vitória (VI) & 7.469 & 1,75 & 17,8 & 6,1 \\
\hline
\end{tabular}

Após ter os dados necessários compilados o próximo passo é calcular a localização horizontal e vertical, possibilitando assim indicar qual o melhor local para implantar o novo centro de distribuição:

a) Localização horizontal:

$(28.124 \times 1,40 \times 10,5)+(9.222 \times 1,90 \times 14,3)+(7.469 \times 1,75 \times 17,9)=12,8$

$(28.124 \times 1,40)+(9.222 \times 1,90)+(7.469 \times 1,79)$

b) Localização vertical:

$(28.124 \times 1,40 \times 2,5)+(9.222 \times 1,90 \times 3,1)+(7.469 \times 1,75 \times 6,1)=3,3$

$(28.124 \times 1,40)+(9.222 \times 1,90)+(7.469 \times 1,75)$

Revista Produção Online. Florianópolis, SC, v. 19, n. 3, p. 896-922, 2019 
Conforme cálculos realizados, as coordenadas do novo centro de distribuição deveriam ser 12,83 e 3,32, ficando dentro do estado de SP, próximo ao porto da cidade de Santos, tal como representado pela linha verde na Figura 9.

D) Método Ponto de Equilíbrio

Para poder ser calculado por este método foi necessário levantar os custos fixos e as variáveis em cada estado. Para os custos fixos foi elaborada a Tabela 5 que traz o custo médio do aluguel para armazenagem nos estados de SP, RJ e ES, considerando a mesma área de armazenagem atual da empresa que é de $2.300 \mathrm{~m}^{2}$ no CD de Caxias do Sul do qual partem todas as entregas para São Paulo. Os demais custos fixos, como energia, água, impostos, não foram considerados pelo fato de não apresentarem fontes seguras e atualizadas, já nos custos variáveis foram utilizados o custo de transporte nos três estados conforme já apresentado na Tabela 4, multiplicado pela média mensal transportada em 2016 que foi de 20.116 toneladas. Como este valor precisa ser calculado por unidade, o resultado foi dividido pela quantidade a ser armazenada mensalmente. Para a quantidade armazenada foi considerada a média mensal armazenada no período de 2012 a 2016, conforme Tabela 6.

Tabela 5 - Custo de aluguel para armazenagem por $\mathrm{m}^{2}$

\begin{tabular}{|c|c|}
\hline Estados & Custo Aluguel por $\mathbf{~}^{\mathbf{2}}$ \\
\hline SP & $R \$ 20,25$ \\
\hline RJ & $R \$ 24,55$ \\
\hline ES & $R \$ 23,22$ \\
\hline
\end{tabular}

Fonte: Colliers International Brasil (2016)

Tabela 6 - Quantidades unitárias armazenadas

\begin{tabular}{cc}
\hline Tabela 6 - Quantidades unitarias armazenadas \\
\hline 2016 & Quantidade armazenada (itens) \\
2015 & 895.724 \\
2014 & 659.442 \\
2013 & 849.305 \\
2012 & 563.824 \\
\hline
\end{tabular}

Fonte: Departamento de logística (2016)

\begin{tabular}{cccc}
\hline $\begin{array}{c}\text { Cidades } \\
\text { Candidatas }\end{array}$ & $\begin{array}{c}\text { Custo Fixo mensal } \\
\text { (R\$) }\end{array}$ & $\begin{array}{c}\text { Custo } \\
\text { Variável/unidade (R\$) }\end{array}$ & $\begin{array}{c}\text { Quantidade } \\
\text { armazenada mensal } \\
\text { (unidades) }\end{array}$ \\
\hline SP & $46.575,00$ & 0,46 & 61.497 \\
RJ & $56.465,00$ & 0,62 & 61.497 \\
VI & $53.406,00$ & 0,57 & 61.497 \\
\hline
\end{tabular}


Os custos totais (CT) são dados por:

$$
\begin{array}{ll}
\mathrm{CT}=\mathrm{CF}+\mathrm{CV} \times \mathrm{Q} & \\
\mathrm{CT} T_{\mathrm{SP}}=46.574,00+(0,46 \times 61.496) & \underline{\mathrm{CTSP}=\mathrm{R} \$ 74.826,60} \\
\mathrm{CT}_{\mathrm{RJ}}=56.465,00+(0,62 \times 61.496) & \underline{\mathrm{C} T_{\mathrm{RJ}}=\mathrm{R} \$ 94.592,52} \\
\mathrm{CTVI}=53.406,00+(0,57 \times 61.496) & \underline{\mathrm{CTVI}=\mathrm{R} \$ 88.458,72}
\end{array}
$$

A localidade mais atrativa, novamente, é a cidade de São Paulo, seguida por Vitória no estado do Espírito Santo, e por último, com o custo mais alto, a cidade do Rio de Janeiro.

\subsection{Dimensionamento do estoque no cd}

\subsubsection{Método da Média Móvel}

Para realizar este cálculo foram utilizados os dados da Tabela 7, que traz as quantidades que foram armazenadas no período de 2012 a 2016. Não foi considerado o volume vendido, pois assim não haveria uma real noção de todo material que fica armazenado, apenas do que foi vendido.

$$
\mathrm{CM}=\frac{721.504+563.824+849.305+659.442+895.724}{5}=737.960 \text { unidades }
$$

Através deste método foi projetado um armazenamento de 737.960 unidades durante o período de um ano, isto mensalmente representaria 61.497 unidades por mês a serem armazenadas.

\subsubsection{Método da Média Móvel Ponderada}

Neste método foi envolvido os gestores da área comercial para atribuir de forma mais correta os pesos a serem atribuídos a cada ano anterior, e as informações de volumes seguem sendo as de número de itens estocados no período de 2012 a 2016, conforme Tabela 7. 
Tabela 7 - Quantidades unitárias armazenadas e peso relativo atribuído

\begin{tabular}{ccc}
\hline Ano & Quantidade (unidades) & Peso relativo \\
\hline 2012 & 721.504 & $5 \%$ \\
2013 & 563.824 & $10 \%$ \\
2014 & 849.305 & $15 \%$ \\
2015 & 659.442 & $25 \%$ \\
2016 & 895.724 & $45 \%$ \\
Total & $\mathbf{3 . 6 8 9 . 7 9 9}$ & $\mathbf{1 0 0 \%}$ \\
\hline
\end{tabular}

Fonte: Elaborada pelo autor (2016)

$$
\begin{aligned}
& \text { DP }(\mathrm{mmp})=(721.504 \times 5)+(563.824 \times 10)+(849.305 \times 15)+(659.442 \times 25) \\
& +(895.724 \times 45)=(78.779 .005) / 100=787.790 \text { unidades }
\end{aligned}
$$

Através do método da média móvel ponderada foi projetado um armazenamento de 787.790 unidades por ano, mensalmente representaria 65.649 unidades.

\subsubsection{Método dos Mínimos Quadrados}

Para utilizar este método primeiramente foi necessário montar a Tabela 8 , utilizando como base as quantidades armazenadas no período de 2012 a 2016 e atribuindo os valores de $\mathrm{x}$ e $\mathrm{y}$.

\begin{tabular}{ccccc}
\multicolumn{5}{c}{ Tabela 8 - Dados para resolução do Método dos Mínimos Quadrados } \\
\hline Ano & $\mathbf{Y}$ & $\mathbf{X}$ & $\mathbf{X}^{2}$ & $\mathbf{X ~ . ~ Y ~}$ \\
\hline 2012 & 721.50 & 0 & 0 & 0 \\
2013 & 563.82 & 1 & 1 & 563.8 \\
2014 & 849.30 & 2 & 4 & 1.698 \\
2015 & 659.44 & 3 & 9 & 1.978 \\
2016 & 895.72 & 4 & 16 & 3.582 \\
Total & $\mathbf{3 . 6 8 9 . 7}$ & $\mathbf{1 0}$ & $\mathbf{3 0}$ & $\mathbf{7 . 8 2 3}$ \\
\hline
\end{tabular}

Onde resultam as equações:
a) $\sum Y=N \cdot a+b \sum x$
3.689.799 $=5 a+10 b$
b) $\quad \sum x y=a \sum x+b \sum x^{2}$
$7.823 .656=10 a+30 b$

Revista Produção Online. Florianópolis, SC, v. 19, n. 3, p. 896-922, 2019 
Resolvendo as duas equações, simultaneamente, isolando "b" ou "a" e substituindo na outra equação, os valores de "a" e "b" obtidos são, respectivamente, 44,4 e 782.232,4. A previsão para 2016 está seis anos à frente de 2012, assim a resolução da equação é:

$$
\begin{aligned}
& y=a+b(x) \\
& y=782.232,4+44,4 \quad(6) \\
& y=782.498,8=782.499 \text { unidades. }
\end{aligned}
$$

A previsão de produtos que deverão ser estocados no ano de 2016 será de 782.449 unidades anuais, ou, 65.204 unidades mensais.

\subsection{Avaliação dos resultados}

Através das técnicas de localização utilizadas foi possível verificar o diferencial de cada um dos locais específicos, proporcionando assim fundamento para a escolha do local definitivo. Para facilitar a avaliação, os dados foram compilados no Quadro 2. O resultado do Método Carga Distância não é apresentado como comparativo nessa figura porque o transporte continuará sendo terceirizado pela empresa. No entanto, o resultado da Tabela 3, recai sobre a opção por São Paulo que apresenta menor lead-time do que as outras localidades, corroborando com a decisão

\begin{tabular}{|c|c|c|c|}
\hline \multirow{2}{*}{ Métodos } & \multicolumn{3}{|c|}{ Local específico } \\
\hline & São Paulo & Rio de Janeiro & Vitória \\
\hline Poderação de fatores & Pontuação de 445 & Pontuação de 355 & Pontuação de 300 \\
\hline Centro de gravidade & Conforme resultado do métoc & $\begin{array}{l}\text { o do centro de gravidade a lo } \\
\text { do estado de São Paulo }\end{array}$ & alização sugerida ficou dentro \\
\hline $\begin{array}{c}\text { Ponto de equilibrio / } \\
\text { custo total }\end{array}$ & Custo Total de $\mathrm{R} \$ 74.826,60$ & Custo Total de $\mathrm{R} \$ 94.592,52$ & Custo Total de $\mathrm{R} \$ 88.458,72$ \\
\hline
\end{tabular}
apontada pelo Quadro 2.

Quadro 2 - Compilação dos resultados das análises

Fonte: Elaborada pelo autor (2016). 
Na ponderação de fatores foram levantados os principais pontos qualitativos, aqueles que não podem ser medidos em moeda. Os que apresentaram maior importância foi a proximidade com o mercado potencial, considerando os principais clientes da empresa e a proximidade com os fornecedores, que neste caso pode-se incluir a facilidade no recebimento dos produtos importados. A cidade de São Paulo apresentou maior escore nesse quesito.

No método do centro de gravidade, além das coordenadas de cada local específico, foi levantado as quantidades movimentadas no ano de 2016 para os estados de SP, RJ e ES e o custo de transporte nestes estados. Após a aplicação do método o resultado indicou como sendo mais favorável para implantação do novo centro de distribuição o estado de São Paulo, mais precisamente perto da cidade de Santos, este método tem como desvantagem o fato do local indicado coincidir com uma região inviável.

Para realização ponto de equilíbrio foi utilizado a média mensal das quantidades armazenadas no período de 2012 a 2016, os custos fixos, custos variáveis resultando no custo total da operação nas três cidades. Desta forma a cidade de São Paulo novamente foi indicada como melhor localização, pois apresentou o menor custo total.

Diante dos resultados dos métodos de análise fica claro que a cidade de São Paulo é a mais indicada para a implantação do novo centro de distribuição, além de estar mais próximo dos principais clientes apresenta a estrutura com menor custo fixo de $\mathrm{R} \$ 46.574,00$, quando comparado com as cidades do Rio de Janeiro e Vitória.

Além dos métodos de localização, também foi estimado a quantidade a ser armazenada no novo CD (Tabela 9), o que demonstrou não haver diferença significativa entre os métodos empregados. Com base no maior valor obtido, 65.649 unidades mensais, foram calculados os custos para manutenção do estoque de produtos na cidade de São Paulo, o que equivale a 788.000 unidades por ano, aproximadamente, e que devem ser movimentadas e entregues para os clientes. 
Tabela 9 - Quantidades a serem mantidas em estoque no novo CD

\begin{tabular}{lcccc}
\hline & Média Móvel & $\begin{array}{c}\text { Métodos de Previsão } \\
\text { Média Móvel Ponderada }\end{array}$ & Mínimos Quadrados \\
\hline $\begin{array}{l}\text { Quantidade } \\
\text { (unidades) }\end{array}$ & mensal & 61.497 & 65.649 & 65.204 \\
\hline
\end{tabular}

Para realizar a comparação da cidade escolhida, São Paulo, com a estrutura atual de armazenagem e expedição em Caxias do Sul foram avaliados os seguintes aspectos:

a) Proximidade com os clientes: a estrutura atual está muito longe dos principais mercados consumidores da empresa, nesse caso a opção por São Paulo proporciona essa vantagem. Estima-se que esta proximidade vai fortalecer a relação com os clientes e possibilitar outras estratégias de ações de vendas, como eventos voltados para a linha de refrigeração e industrial nas principais lojas, maior divulgação da linha de produtos entre outros.

b) Tempo de entrega dos pedidos: a estrutura atual por estar na extremidade Sul do Brasil apresenta desvantagens em relação aos concorrentes quando se trata de tempo de entrega dos matérias adquiridos pelos clientes. Em uma comparação simples se for pego a cidade de Manaus como sendo destino hoje a entrega ficaria em até 25 dias úteis. Com o novo $C D$ em São Paulo este prazo reduziria para 18 dias úteis, conforme Figura 8, destino AM (estado do Amazonas).

c) Custo de transporte: conforme dados fornecidos pela empresa no ano de 2016 foi gasto mensalmente em torno de $\mathrm{R} \$ 28.000,00$, este considerando uma média mensal de 20.116 toneladas transportadas por mês, que equivale a $R \$ 1,39$ por tonelada. Considerando o mesmo volume transportado de 20.116 toneladas por mês com o valor de frete de São Paulo de $\mathrm{R} \$ 1,40$ por tonelada, resultaria no valor médio de $R \$ 28.162,40$ mensais. Nestas duas simulações não foi levado em conta as distâncias percorridas para realizar as entregas dentro de São Paulo. Como São Paulo está mais próximo dos principais mercados consumidores a tendência é que o custo com transporte dentro de São Paulo seja menor do que na estrutura atual, partindo de Caxias do Sul e fazendo entregas em cada destino.

d) Custo com aluguel: atualmente o custo com aluguéis, em Caxias do Sul, é de $\mathrm{R} \$ 20.000,00$ por mês, se fosse locada a mesma área na cidade de São Paulo o custo seria de $\mathrm{R} \$ 46.575,00$ por mês, um valor bem superior ao que é pago com a 
estrutura atual em Caxias do Sul. Dentre os pontos analisados este foi o único que apresentou desvantagem econômica.

\section{CONSIDERAÇÕES FINAIS}

A questão de pesquisa que orientou esse trabalho foi verificar qual a melhor opção para instalação de um novo centro de distribuição. Essa questão foi satisfatoriamente respondida através dos métodos de análise e comparação. Os principais ganhos com a alternativa escolhida que envolve a instalação em São Paulo são a redução dos prazos de entrega dos pedidos, redução dos custos fixos anuais da empresa com a terceirização da armazenagem e expedição com um agente logístico estimada em $R \$ 46.143,00$ e redução nos custos com frete, estimada em $15 \%$. O custo total da terceirização será em torno de $\mathrm{R} \$ 275.800,00$ por ano.

A melhor opção para a empresa é manter o centro administrativo na cidade de Caxias do Sul com um pequeno estoque para atender as demandas da loja física, clientes da região e filiais, e terceirizar o maior volume com algum operador logístico na cidade de São Paulo. Orçamentos realizados com duas empresas apontaram que o valor médio cobrado é de $\mathrm{R} \$ 0,30$ para operações logísticas que incluem recebimento, armazenagem e expedição, mais $R \$ 0,05$ de seguro, totalizando $R \$ 0,35$ por unidade. Se for considerada a quantidade estimada de 788.000 unidades para armazenamento no ano de 2016, o valor gasto seria de $R \$ 275.800,00$ anuais. Conforme informações da empresa, atualmente é gasto somente em aluguel o valor de $R \$ 240.000,00$ por ano, se for levado em consideração os demais gastos com luz, manutenção predial e de equipamentos, funcionários e outros encargos o valor ultrapassa $R \$ 320.000,00$. Desta forma fica evidente que a terceirização é a melhor alternativa para a abertura deste novo CD, possibilitando à empresa utilizar o capital disponível para aplicar em áreas que tragam maior retorno. Entre os principais ganhos com a implantação terceirizada em São Paulo pode-se citar:

a) Redução nos custos com alugueis, energia elétrica, manutenção e conservação do armazém, aquisição de equipamentos como empilhadeiras e porta pallets, além de salários e encargos com funcionários. 
b) Visão dos processos, através da terceirização da armazenagem será possível acompanhar o processo como um todo, ficando mais fácil detectar falhas e consequentemente desenvolver medidas para aperfeiçoar o serviço. À medida que os processos forem otimizados é possível ganhos na agilidade, assertividade, culminando também em redução de custos.

c) Aumento no retorno do investimento, com a terceirização do processo de armazenagem os gestores da empresa terão mais tempo para o planejamento da empresa, como inclusão de novos processos ou até mesmo de novas áreas que auxiliem no desenvolvimento e ampliação do negócio, tornando a empresa mais competitiva;

d) Maior eficiência, com a gestão do processo de armazenagem sendo realizada por profissionais altamente qualificados e especializados no assunto, a empresa conseguirá maximizar sua capacidade efetiva, uma vez que a eficiência na hora de receber, armazenar e expedir terá um aumento significativo;

e) Segurança e qualidade, os armazéns terceirizados geralmente possuem localização privilegiadas, próximos a estradas que facilitam o acesso a grandes centros, contam também com certificações como a ISO 9001 o que gera ainda maior confiabilidade nos processos realizados, além de possuir itens de segurança do espaço e das mercadorias estocadas;

f) Satisfação dos clientes, não menos importante que os demais. Ao terceirizar o serviço todo o processo de recebimento, armazenagem e carregamento dos pedidos serão realizados de forma mais rápida e eficaz, aprimorando assim o atendimento aos clientes, reduzindo também as reclamações de envios de produtos errados, melhorando a qualidade dos serviços prestados e ganhando vantagem competitiva.

Gaither e Frazier (2002) defendem que a terceira parte da logística (3PL), na figura dos operadores logísticos, pode fornecer o que há de mais moderno em sistemas de informação logística, menores preços negociados para transportadores e espaço de armazenamento, bem como, pessoal de logística, altamente treinado.

Os métodos aqui utilizados para localização do novo CD, Método do Centro de Gravidade, Método do Ponto de Equilíbrio, Método Carga-Distância e Método de Ponderação de Fatores, são clássicos, estão consolidados na literatura e são 
relativamente fáceis de serem compreendidos e aplicados. A confiabilidade de seus resultados depende da precisão em obter os dados numéricos de quantidades armazenadas e movimentadas e dos custos envolvidos nessas atividades. Como a empresa aqui estudada é experiente na importação de itens e de suas comercializações no mercado interno, os dados numéricos necessários para os cálculos tem um bom poder de aproximação, reduzindo a margem de erro da decisão pela cidade de São Paulo e pela terceirização da operação logística. Métodos multicritérios, como o AHP (Analytic Hierarchy Process), também são utilizados para decisões de localização (Briozo e Musetti, 2015), principalmente, quando o número de agentes envolvidos no processo de tomada de decisão, o número de variáveis, a presença de discrepância de opiniões e de subjetividade, for maior. Nesse estudo de caso, a decisão recai principalmente sobre os dois diretores que compartilham das mesmas opiniões e possuem conhecimento detalhado e dados controláveis de suas operações. Dessa forma, métodos clássicos envolvendo variáveis conhecidas, quantificadas e que podem ser estimadas com relativa facilidade pelos seus poucos agentes, e ainda, considerando que o problema não é de grande complexidade, como nesse artigo, tais métodos podem ser uma boa prática para direcionar a tomada de decisão sobre a localização de novas unidades de um negócio já existente.

\section{REFERÊNCIAS}

BRIOZO, R. A.; MUSETTI, M. A. Método multicritério de tomada de decisão: aplicação ao caso da localização espacial de uma Unidade de Pronto Atendimento - UPA 24 h. Gestão e Produção, São Carlos, v. 22, n. 4, p. 805-819, 2015. https://doi.org/10.1590/0104-530X975$\underline{13}$

CECCATO, C.; BELFIORE, P. O uso de métodos de previsão de demanda nas indústrias alimentícias brasileiras. Gestão e Produção, São Carlos, v. 22, n. 2, p. 404-418, 2015. https://doi.org/10.1590/0104-530X108-12

CORRÊA, H. L. Administração de cadeias de suprimento e logística: o essencial. Atlas, São Paulo: Atlas, 2014.

CORRÊA, H. L.: CORRÊA, C. A. Administração da produção e operações, manufatura e serviços: uma abordagem estratégica. São Paulo: Atlas, 2012.

DIAS, M. A. P. Administração de materiais: princípios, conceitos e gestão. São Paulo: Atlas, 2012.

DIEHL, A. A. Pesquisa em ciências sociais aplicadas: métodos e técnicas. São Paulo: Prentice Hall, 2004. 
GAITHER, N.; FRAZIER, G. Administração da produção e operações. São Paulo: Pioneira, 2002.

GONÇALVES, P. S. Logística e cadeia de suprimentos: o essencial. São Paulo: Manole, 2013.

GRANT, D. B. Gestão de logística e cadeia de suprimentos. São Paulo: Saraiva, 2013.

GUAZZELLI, C. S.; CUNHA, C. B. Otimização multicritério para o problema de localização de centros de distribuição de uma empresa com unidade produtiva no Polo Industrial de Manaus. Gestão e Produção, v. 22, n. 3, p. 480 - 494, 2015. https://doi.org/10.1590/0104$\underline{530 \times 1980-15}$

GURGEL, F. A. Logística industrial. São Paulo: Atlas, 2008.

HEIZER, J.; RENDER, B. Administração de operações: Rio de Janeiro: LTC, 2001.

KRAJEWSK, L. J.; RITZMAN, L. J.; MALHOTRA, M. Administração de produção e operações. São Paulo: Pearson Prentice Hall, 2009.

KUSSANO, M. R.; BATALHA, M. O. Custos logísticos agroindustriais: avaliação do escoamento da soja em grão do Mato Grosso para o mercado externo. Gestão e Produção, v. 19, n. 3, p. 619-632, 2012. https://doi.org/10.1590/S0104-530X2012000300013

MOREIRA, D. A. Administração da produção e operações. São Paulo: Cengage Learning, 2008.

NOGUEIRA, A. S. Logística empresarial: uma visão local com pensamento globalizado. São Paulo: Atlas, 2012.

PAOLESCHI, B. Estoques e armazenagem. São Paulo: Érica, 2014.

RITZMAN, L. P.; KRAJEWSKI, L. J. Administração da produção e operações. São Paulo: Prentice Hall, 2004.

ROESCH, S. M. A. Projetos de estágio e de pesquisa em administração: guia para estágios, trabalhos de conclusão. São Paulo: Atlas, 2013.

RUSSO, C. P. Armazenagem, controle e distribuição. Curitiba: Intersaberes, 2013.

TAYLOR, D. A. Logística na cadeia de suprimentos: uma perspectiva gerencial. São Paulo: Pearson, 2005.

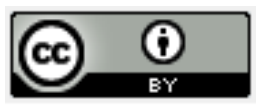

Artigo recebido em: 29/07/2018 e aceito para publicação em: 16/08/2019

DOI: http://dx.doi.org/10.14488/1676-1901.v19i3.3330 